\title{
CoDNaS-RNA: a database of Conformational Diversity in the Native State of RNA
}

Martín González Buitrón ${ }^{1}$, Ronaldo Romario Tunque Cahui², Emilio García Ríos², Layla Hirsh $^{2}$, María Silvina Fornasari ${ }^{1,3}$, Gustavo Parisi ${ }^{1,3}$, Nicolas Palopoli ${ }^{1,3, *}$

${ }^{1}$ Departamento de Ciencia y Tecnología, Universidad Nacional de Quilmes, Buenos Aires, Argentina, ${ }^{2}$ Departamento de Ingeniería, Pontificia Universidad Católica del Perú, Lima, Perú, ${ }^{3}$ Consejo Nacional de Investigaciones Científicas y Técnicas, Buenos Aires, Argentina.

*To whom correspondence should be addressed.

\begin{abstract}
Conformational changes in RNA native ensembles are central to fulfill many of their biological roles. Systematic knowledge of the extent and possible modulators of this conformational diversity is desirable to better understand the relationship between RNA dynamics and function.

We have developed CoDNaS-RNA as the first database of conformational diversity in RNA molecules. Known RNA structures are retrieved and clustered to identify alternative conformers of each molecule. Pairwise structural comparisons within each cluster allows to measure the variability of the molecule. Additional data on structural features, molecular interactions and functional annotations are provided. CoDNaS-RNA is implemented as a public resource that can be of much interest for computational and bench scientists alike.
\end{abstract}

Availability: CoDNaS-RNA is freely accessible at http://ufq.unq.edu.ar/codnasrna

Contact: npalopoli@unq.edu.ar

\section{Introduction}

Many macromolecules alternate between dynamic conformational states to carry out their biological functions. For RNAs in particular, studying this ensemble of conformers is essential to gain the most thorough description of their native state (Ganser et al., 2019). The rugged nature of the RNA energy landscape enables functionally relevant conformational changes (Simon and Gehrke, 2009), not only associated with the inherent physicochemical properties of the nucleotide chain, but also triggered by external factors such as changes in temperature or $\mathrm{pH}$ and interactions with proteins, metals or small ligands (Mustoe et al., 2014). These structural transitions, ranging from local base-pairing perturbations to long-distance domain rearrangements, play a central role in RNA activities such as self-induced folding during transcription, the catalytic activity of ribozymes, the response to 
altered cellular conditions by riboswitches and the assembly of ribonucleoproteins (Al-Hashimi and Walter, 2008).

In general, structural molecular biology databases do not address the native ensemble of their entries. Some of the many protein structure databases consider conformational diversity explicitly, namely CoDNaS, a collection of alternative tertiary structures of proteins developed in our group (Monzon et al., 2016) and PDBFlex (Hrabe et al., 2016). While there are databases that compile structural information of RNAs, including valuable resources such as Rfam (Kalvari et al., 2018) and URS Database (Baulin et al., 2016), these seem to be fewer, less actively maintained or without an easy and comprehensive access to data compared with their protein counterpart. To our knowledge, none of these RNA data resources address conformational diversity explicitly.

In this context, we have developed CoDNaS-RNA to fill a gap in bioinformatics resources to study RNA structure. CoDNaS-RNA constitutes the first RNA database that integrates structural data and annotations to highlight conformational diversity as an essential feature to understand RNA dynamics and function.

\section{Dataset construction}

Each entry in CoDNaS-RNA compiles a cluster of known structures of RNAs with the same sequence, as determined in separate experiments and possibly under different conditions (Figure 1A). Consequently, these conformers can be considered as alternative instances of the RNA structure in its native ensemble. Structural information is obtained directly from mmCIF files in the Protein Data Bank (Berman et al., 2000). RNA sequence clusters are built with CD-HIT (at 100\% identity and 98\% coverage) (Li and Godzik, 2006) and validated with Blastclust (National Center for Biotechnology Information (NCBI). Documentation of the BLASTCLUST-algorithm.) for a robust identification of alternative conformers of the same RNA. All-vs-all pairwise structural comparisons are calculated in each cluster with TMalign (Zhang and Skolnick, 2005). The extent of observed conformational diversity in each RNA is mainly assessed by the maximum RMSD among any given pair of its conformers, with metrics such as TM-score also informed. DSSR (Lu et al., 2015) is used to extract all intraand inter-chain contacts for these conformers, allowing for the comparison of interaction interfaces. External data about ncRNAs is cross-referenced from RNAcentral (The RNAcentral Consortium, 2019) to facilitate further exploration of relevant features. CoDNaS-RNA is first released with 1000 clusters of RNA structures from 2208 out of 3057 validated PDBs ( $\sim 77 \%$ of available PDB entries), comprising an average of 9.5 conformers per cluster.

\section{Usage}

CoDNaS-RNA can be searched by one of several criteria (Figure 1B), such as the internal cluster identifier, PDB code, RNA type or name, organism or taxon number, Unique RNA 
Sequence (URS) identifier, and title or abstract of original publication. Once a cluster is selected from all matching entries, the website provides detailed data on the cluster, organized as separate sections (Figure 1C):

- General information about the cluster e.g. sequence length, cluster sequence identity and source organism. One or more stable URS are assigned to the cluster based on mapping to RNAcentral. The same external resource provides annotations of RNA types.

- Structural information. Provides global statistics from pairwise structural comparisons between members of the cluster. The number of conformers showcases the available evidence on conformational diversity in the cluster. The average, minimum and maximum RMSD and TMscore values, provide the central measurements of conformational diversity.

- Structural overview of conformers, with the full 3D structure for each PDB entry and chain (conformer) in the cluster. These views are presented along with brief structural information and details on the primary citation of the PDB file.

- Dendrogram summarizing the structural clustering of all conformers. Obtained by performing complete linkage hierarchical clustering on pairwise RMSD values.

- Information about the pair of conformers with maximum conformational diversity, i.e. a pair of conformers that display the maximum RMSD value between them. Displays a table of individual and compared data, mainly cross-referenced from the RCSB PDB and taken from DSSR interaction features.

- Interactions on the pair of conformers with maximum conformational diversity. Provides details on the total number of interactions per conformer, presented separately for intra-chain contacts and two inter-chain types of contacts: with other nucleotides and with proteins.

- Sequence information. Shows the sequences of the pair of conformers with maximum RMSD between them, which may differ if at least one is truncated or mutated.

- Interactive view of the structural superposition of conformers with maximum conformational diversity.

The complete datasets used by CoDNaS-RNA can be downloaded as a tab-separated file available from http://ufq.unq.edu.ar/codnasrna/data/codnasrna v1.0.0.tar.gz

\section{Conclusions}

CoDNaS-RNA is a unique, easy-to-use database to gather information on RNA native ensembles and inspect their conformational diversity. The database showcases possible associations between the diversity in the native ensemble and physicochemical, biological or functional modulators such as origin of the RNA, $\mathrm{pH}$ or temperature and binding to ligands. Interaction data is provided at residue level, with at least one conformer in $66 \%$ of RNA clusters determined with presence of proteins. CoDNaS-RNA can help to discriminate synthetic conformers from natural ones, while still informing about their shared properties. 
Moreover, CoDNaS-RNA may also facilitate annotation of RNAs of unspecific types by allowing prospective curators to inspect the annotations of alternative conformers in the cluster of interest. We foresee CoDNaS-RNA can become an essential resource for the wide community of computational and bench scientists.

\section{Funding}

This work has been supported by grants from Universidad Nacional de Quilmes (PUNQ 1309/19), Agencia Nacional de Promoción de la Investigación, el Desarrollo Tecnológico y la Innovación (PICT-2018 3457) and Consejo Nacional de Investigaciones Científicas y Técnicas (CONICET) (PIP-2015-2017 11220150100853CO) from Argentina. M.S.F., G.P. and N.P. are Researchers from CONICET.

\section{References}

Al-Hashimi,H.M. and Walter,N.G. (2008) RNA dynamics: it is about time. Curr. Opin. Struct. Biol., 18, 321-329.

Baulin,E. et al. (2016) URS DataBase: universe of RNA structures and their motifs. Database (Oxford), 2016.

Berman,H.M. et al. (2000) The protein data bank. Nucleic Acids Res., 28, 235-242.

Ganser,L.R. et al. (2019) The roles of structural dynamics in the cellular functions of RNAs. Nat. Rev. Mol. Cell Biol., 20, 474-489.

Hrabe,T. et al. (2016) PDBFlex: exploring flexibility in protein structures. Nucleic Acids Res., 44, D423-8.

Kalvari,I. et al. (2018) Rfam 13.0: shifting to a genome-centric resource for non-coding RNA families. Nucleic Acids Res., 46, D335-D342.

Li,W. and Godzik,A. (2006) Cd-hit: a fast program for clustering and comparing large sets of protein or nucleotide sequences. Bioinformatics, 22, 1658-1659.

Lu,X.-J. et al. (2015) DSSR: an integrated software tool for dissecting the spatial structure of RNA. Nucleic Acids Res., 43, e142.

Monzon,A.M. et al. (2016) CoDNaS 2.0: a comprehensive database of protein conformational diversity in the native state. Database (Oxford), 2016.

Mustoe,A.M. et al. (2014) Hierarchy of RNA functional dynamics. Annu. Rev. Biochem., 83, $441-466$.

National Center for Biotechnology Information (NCBI). Documentation of the BLASTCLUST-algorithm. .

Simon,A.E. and Gehrke,L. (2009) RNA conformational changes in the life cycles of RNA viruses, viroids, and virus-associated RNAs. Biochim. Biophys. Acta, 1789, 571-583.

The RNAcentral Consortium (2019) RNAcentral: a hub of information for non-coding RNA sequences. Nucleic Acids Res., 47, D221-D229.

Zhang, Y. and Skolnick,J. (2005) TM-align: a protein structure alignment algorithm based on the TM-score. Nucleic Acids Res., 33, 2302-2309. 


\section{Figures}

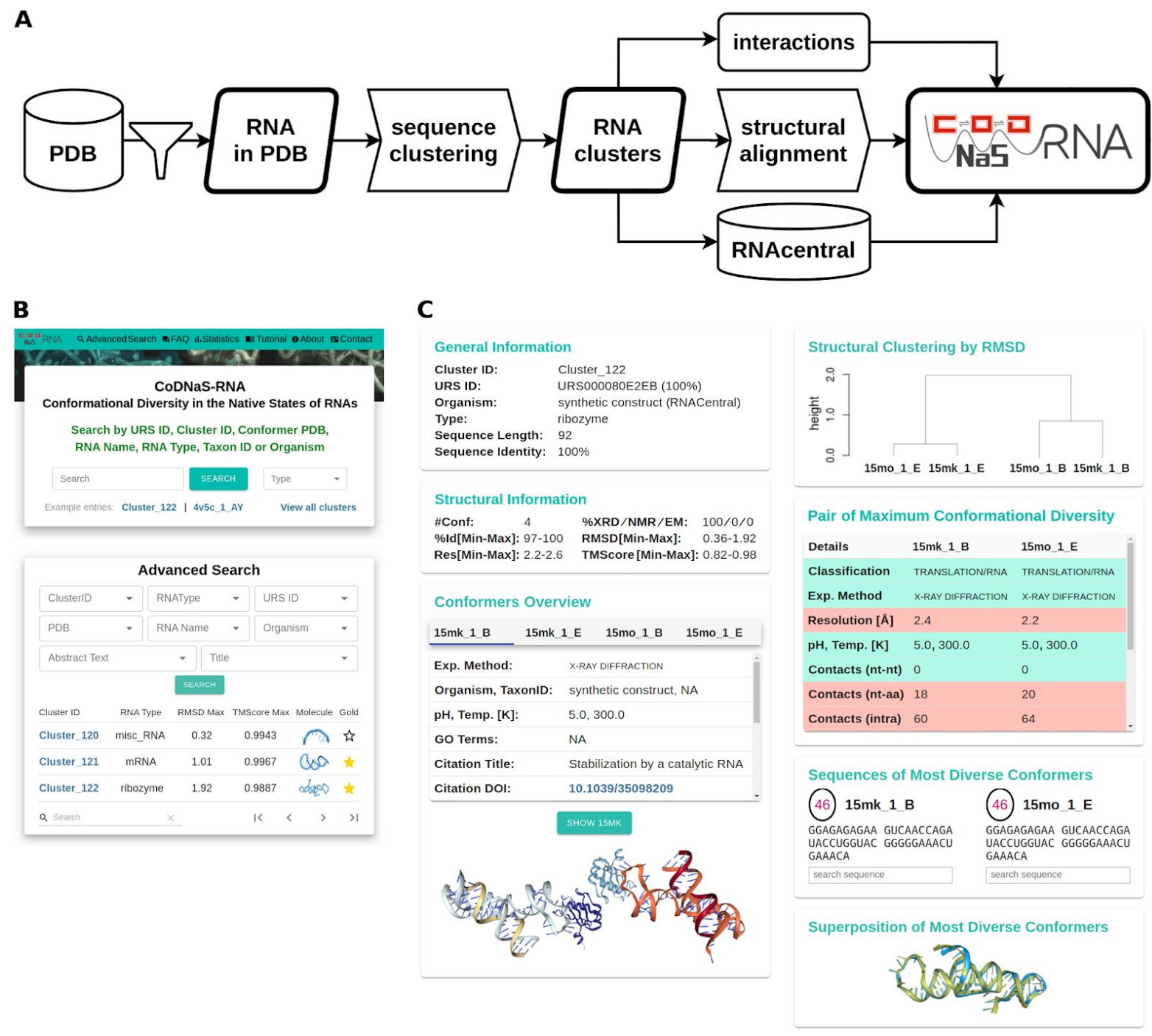

Figure 1. Panel A: Schematic workflow for data acquisition and processing to build CoDNaS-RNA. See details in main text. Panel B: Overview of the basic and advanced search interfaces in CoDNaS-RNA. Panel C: Overview of the main information provided for any selected entry in CoDNaS-RNA. From top to bottom, left column: General information about the selected RNA; structural information about the available RNA conformers and their comparison: and general description of individual confomers. From top to bottom, right column: dendogram of the RMSD-based hierarchical clustering of available conformers; structural comparison of the pair of conformers displaying the maximum conformational diversity as measured by the RMSD between them; searchable sequences of these two confomers; and structural superposition of these two conformers. Panels B and C only show some of the available options and do not necessarily match real entries in the database. 